\title{
Opportunities and Challenges in Paper Printed RFID
}

\author{
Shalini Jain \\ Marathwada Institute of Technology, Dr. Babasaheb Ambedkar Marathwada University, India \\ Abhinav Palia \\ University of Southern California Viterbi School of Engineering, California, USA
}

\begin{abstract}
RFID technique has gathered interest in many areas. But due to high installation cost and comparatively low read range, it has not reached its full potential. In this paper, how 'conductive' ink-jet printing technique, as an effective approach for the printing of RFID tags on paper has been studied. Various results characterize paper as a good substrate for the fabrication of sensor modules. Also, the use of implantable RFID tags for bio-monitoring applications is investigated that may open interesting opportunities in telemedicine. Although the technique is exciting enough to be adopted and can revolutionize data fusion but still there are certain unresolved issues that can restrict its use for the development of a 'ubiquitous' network for device and body monitoring applications.
\end{abstract}

Key words: $\quad$ CNT, Micro-Strip Ring Resonator, RFID, Tissue Mimicking, Wearable Applications.

\section{Introduction}

Radio Frequency Identification (RFID) is an emerging compact wireless technology for identification of objects using radio waves. It involves the use of interrogators (known as readers) and tags (known as labels). Tags are of two types- Passive tags and Active tags. In case of passive tags, reader continuously emits RF carrier signals which are received by the tags, modifies it and retransmits it. The reflected waves are detected by the reader. On the other hand, active tags are equipped with the battery to generate the carrier theselves[1].

There are many applications of RFID that establish the requirement for the production of large amount of tags at low cost. The idea of using environment friendly organic substrates for making tags seems to be an attractive alternative over the commonly used metal antennas, that have a limited

range, prone to corrosion, not flexible, heavy in weight and high in cost. Also due to the dielectric between the metal antenna material and mostly the ionized water of human body, the near field gets disturbed. Even wearable bracelet-like sensing devices have a very low range and are not much successful due to these factors.

Paper, one of the easily available organic substrate, along with its advantages that it is low cost, can be made hydrophobic, fire retardant, and its dielectric characterization makes it an eminent candidate for the realization of the RFID tags. Inkjet printing with conductive ink can be used for the fabrication of circuits.
It is a better method than the usual etching method that involves removal of unwanted metal from the substrate, whereas while printing, single ink droplet is jetted to the desired position. Thus, causing no waste and more tags can be produced at faster rate.

The concept of implantable RFID tags, is a revolutionary technique for bio-monitoring applications. This has possible uses for human health monitoring, pet tracking and security purposes. However, implantable tags have become a controversial issue in spite of its tremendous use in medical field. Various researches carried out on this technology, motivations and possible challenges to be met are discussed ahead in this paper.

\section{Related Work}

The dielectric characterization of paper, involving the study of its relative permeability as well as the loss tangent (used for determining the amount of loss of signal in the medium) was done using microstrip ring resonator method[1] at the UHF band. The measured results showed a good agreement with the simulations, making it appropriate for developing tags for RFID applications. Now the printing of circuits was the task to be accomplished. Inkjet printing method, that is fast and economical in the sense that no waste is created, can be used to print electronics on organic substrates. The conductive ink composed of Single walled carbon nanotubes(SWCNT's), is used for printing the circuits. CNT network formed by multi- 


\section{Yinternational Research Journa}

p-ISSN 2202-2821 e-ISSN 1839-6518 (Australian ISSN Agency)

dropping at the same spot and its electrical characteristics were studied. The electrical properties of the printed conductive line, impedance measurement were measured and I-V curves were drawn[2]. Further, sintering process is required for removing excess solvent and material impurities from the depositions. As paper printed tags were flexible in nature, its radiation pattern was studied to explore the limits of design. The paper printed tag showed a good performance when bend to form wearable tag with respect to the straight tags, proving this approach could function as an enabling technology for much faster fabrication. Another application of interest was the fabrication of CNT based sensor for wireless sensing of toxic gas, that employed the principle of impedance change of SWCNT coming in contact with the gas[3][4]. the backscattered signal was used by the reader to sense the presence of gas.

Due to high dielectric and conductivity contrast with respect to most parts of the human body, the range of most of the wireless sensors operating on RF and microwave frequencies was limited when attached to the body. This led to the concept of implantable antennas implemented by encapsulating the liquid antenna in flexible plastic containers. The radiation pattern of wearable bracelet liquid antenna, mounted on tissue mimicking dielectric phantom model, were investigated[5]. Also, the sensing capabilities, the backscattering contrast and the communication link were analyzed considering a specific medical application.

Concluding the whole research, we can say that various properties of paper offer it as a useful substrate for printing of circuits using conductive ink - consisting of nanoparticles through inkjet printing process. Using liquid antennas for implantable purposes with better range, also seems to be an effective approach

\section{Future Scope}

Wireless technology, wireless devices have invaded almost in every field. Following the same trend, RFID tags in product promise to go well beyond those already achieved with barcodes as it cannot be replicated and therefore, increase the security of the product. RFID tags can store data upto $2 \mathrm{~Kb}$. Tracking of inventory in a warehouse is one of its major uses. Other application areas include- retail supply chain, military supply chain, pharmaceutical tracking and management access control, sensing and metering applications, parcel and document tracking, automatic payment solution, asset tracking, real time location system (RTLS), automatic vehicle identification, livestock or pet tracking. All these applications

have attracted global attention and many firms are already using it in their business processes
Vol. 07 No. 022017

82800702201701

The effective integration of RFID's in biosensors on biocompatible organics would allow effective realization of BodyArea-Network fully linking both wearable and implantable devices. It would help to collect information about the patient's state during surgery follow up. Also, there is much scope in establishing high security networks, for instance, in keeping track of criminal activities in jail.

However, analysis show that these systems are in a young stage and the technique has not yet reached its full potential. Paper printed tags seem to be a "lighted candle" to overcome the high cost factor so that they can be used everywhere for identification of objects. There are still certain unresolved issues to be considered and are discussed further.

\section{Challenges}

\section{A. Life Safety}

Bio-monitoring devices using RFID have one major disadvantage- tags implanted inside the human body would receive radio waves (electromagnetic in nature), which may be intense enough to cause heating up of the body if received for a long time. The extreme example is what happens when meat is kept in a microwave oven Imagine! Various dangerous reactions might get triggered if a person is continuously being monitored. Prolonged absorption can cause change in behavior, mental imbalance and tissue damage.

\section{B. Ethical Issues}

Ethical issues concerned with the implantable devices are to be considered. No doubt these chips can be seen as potential security devices and emergency solutions, but there is a darker side with erosion of privacy and breach in the bodily integrity. For example, with growing needs of identification, top level secret agencies such as CID, may have identity cards implanted directly in the body of its employees. The scenario would be like $24 \times 7$ slaves with their personal life at risk.

\section{Cost Factor}

The process of inkjet printing can be explained as under-

i. Manufacturing of high quality paper with various textiles added to it to make it hydrophobic, fire retardant, with proper texture etc.

ii. While printing, achievement of curing temperature is required. At lower curing temperature, larger gaps exist between the particles, resulting in poor connection. Thus, heating of substrate is to be done.

iii. Care should be taken while printing i.e. drop needs to be jetted at the right time, surface conditions, drop velocity, and form must be correct otherwise drop will 
splash on the substrate yielding poor quality, further, if a drop is jetted on wrong place, it might cause an open or short circuit.

iv. The time duration for printing using conductive ink is quite high[7].

Clearly, the whole process is complex, needs continuous monitoring, so cannot produce tags at low cost.

\section{Limited Use}

The range of active RFID tags is more than passive tags. But these active tags require some source or battery for their operation. However, only passive tags are designed on organic substrate. This greatly limits its use for certain applications. .

\section{References}

[1]. K. Finkenzellar, RFID Handbook, Wiley, 2004.

[2]. Li. Yang, Amin Rida, Rushi Vyas and MM. Tentzeris, "RFID Tag and RF Structures on a Paper Substrate Using Inkjet-Printing Technology” IEEE Trans. MTT, Vol.55, No.12, Part 2, December 2007.

[3]. Jin-Won Song, Joondong Kim, Yeo-Hwan Yoon, Byung-Sam Choi, Jae-Ho Kim and Chang-Soo Han, "Inkjet printing of single-walled carbon nanotubes and electrical characterization of the line pattern" IOP publishing 2008.

[4]. C. Contalenia, 1. Valentine, 1. Lozzic, I. Armentano, J. M Kenny, L. Lozzi and S.santucci, "Carbon nanotubes as new materials for gas sensing applications”, J.Eur Ceram.Soc, Vol. 24, 2004.

[5]. J-H Yun, H. Chang-Soo, J.Kim, J-W. Song,D.-h. Shin, Y.-G. Park, "Fabrication of carbon nanotube sensor device by inkjet printing", 2008 Proc. of IEEE Nano/Micro engineered and molecular system,2008.

[6]. A. Traille, L. Yang, A. Rida and M.M. Tentzeris, "A novel Liquid Antenna for Wearable Biomonitoring Applications", Procs. of the 2008 IEEE-Ims Symposium, June 2008.

[7]. L.Yang, L.J. Martin, D.Staiculescu, C.P. Wong and M.M. Tentzeris,"Conformal Magnetic Composite RFID for wearable RF and bio monitoring applications", IEEE Trans. MTT, vol.56, December 2008.

[8]. Matti mantysalo, Ville Pekkanen, Kimmo Kaija,Juha Niittynen, Santtu Koskinen, Eerik Halonen, Pauliina Mansikkamaki and Ossi Hameenoja,"Capability of Inkjet Technology in Electronics Manufacturing", Electronic Components and Technology Conference 2009. 